\title{
Equivalentes ventilatorios y pulso de oxígeno máximos en la fase precompetitiva de deportistas militares. (Un estudio observacional)
}

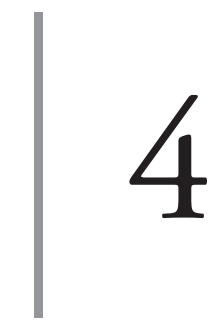

https://doi.org/10.21830/9789585241466.04

\author{
Ana Isabel García Muñoz \\ Hassan Ali Gazwi ${ }^{2}$ \\ Zainab Abdulla Al Robeh ${ }^{3}$
}

\section{Resumen}

Objetivo: analizar los equivalentes ventilatorios para oxígeno $\left(\mathrm{VE} / \mathrm{VO}_{2}\right)$ y el pulso de oxígeno máximos, precompetencia, en un grupo de deportistas militares. Materiales y métodos: estudio retrospectivo que analizó por medio de ergoespirometría el pulso de oxígeno el $\mathrm{VE} / \mathrm{VO}_{2}$ y el dióxido de carbono $\left(\mathrm{VE} / \mathrm{VCO}_{2}\right)$ en relación con otras variables fisiológicas. Se aplicó estadística descriptiva. La homogeneidad de la varianza se evaluó mediante la prueba de Levene. La distribución de los datos se verificó mediante la prueba de Shapiro-Wilk. Se aplicó la T de Student y el coeficiente de Pearson. Resultados: sesenta deportistas con edad $21 \pm 2$ años, $80 \%(n=48)$ hombres y $20 \%(n=12)$ mujeres, ingresaron al estudio. Los equivalentes ventilatorios máximos no mostraron diferencias por género: $p(>0,05)$, pero sí por modalidad deportiva: $p(0,02)$. El pulso de oxígeno máximo evidenció diferencias por género y tipo de deporte: $p(>0,01)$. El $\mathrm{VE} / \mathrm{VCO}_{2 \text { máx. }}$ y el consumo de oxígeno $\left(\mathrm{VO}_{2 \text { máx. }}\right.$ ) se relacionaron con la duración de la prueba, y el pulso de oxígeno máximo con la velocidad. Conclusiones: la eficiencia

1 Terapeuta respiratoria, especialista en rehabilitación cardiopulmonar. Centro de Investigación de la Cultura Física (Cicfi), Escuela Militar de Cadetes "General José María Córdova". Bogotá, Colombia. Contacto: ana.garcia@esmic.edu.co - http://orcid.org/0000-0003-4455-4534

2 Instructor clinico, lider de grupo. Terapeuta respiratorio Senior del departamento de atencion respiratoria en el complejo medico Dammam en Arabia Saudita. Contacto: halgazwi@moh.gov.sa https://orcid.org/0000-0001-6149-4700.

3 Enfermera jefe. Especialista Senior en enfermeria del departamento de enfermeria en el complejo medico Dammam en Arabia Saudita. Contacto: zalrobeh@moh.go.sa - https://orcid.org/00000002-8356-2687. 
ventilatoria para dióxido de carbono y el pulso de oxígeno máximos deben formar parte del entrenamiento físico militar y se debe diferenciar por género y tipo de deporte.

Palabras clave: Ergoespirometría, deporte, $\mathrm{VE} / \mathrm{VCO}_{2 \text { máx., }}$, equivalentes ventilatorios, pulso de oxígeno.

\section{Introducción}

La prueba de ejercicio cardiopulmonar (Pcpe) o ergoespirometría, se define como un procedimiento diagnóstico de la función y la capacidad del sistema cardiovascular, pulmonar y metabólico, que proporciona información sobre la respuesta del cuerpo al estrés dinámico, de tal manera que se utiliza de forma rutinaria en los laboratorios de pruebas de esfuerzo (1). Las funciones respiratorias y el análisis de gases durante la actividad física, al mismo tiempo que la función cardíaca bajo carga, se monitorean y evalúan. La Pcpe es una herramienta de diagnóstico y pronóstico que se utiliza para evaluar la causa de la falta de aliento (disnea) y para proporcionar la evaluación pronóstica de pacientes con enfermedades cardiovasculares y pulmonares, como enfermedad coronaria, enfermedad pulmonar obstructiva crónica (Epoc), embolia pulmonar y síndrome de hiperventilación, así como el asma inducida por el ejercicio, entre otros $(2,3)$.

Es un método no invasivo y objetivo que proporciona una evaluación muy precisa de la función de los sistemas cardiovascular, pulmonar, muscular y metabólico en forma cuantificada durante el esfuerzo. Esto se calcula comúnmente a partir de la velocidad de trabajo de la cinta de correr o del cicloergómetro. Se considera un estándar de oro para la evaluación funcional cardiopulmonar, por lo tanto numerosas especialidades médicas se han beneficiado de esta prueba, como cardiología, neumología y medicina deportiva o ciencia del deporte (4-7).

La Pcpe es un importante método de evaluación funcional en Colombia y en todo el mundo. En sus aplicaciones más frecuentes, consiste en aplicar un ejercicio de intensidad que aumenta gradualmente hasta el agotamiento o hasta la aparición de síntomas y / o signos limitantes (8). La prueba se basa 
en la medición de gases espirados durante el ejercicio, a partir de lo cual se estima, entre otros y de manera directa, la ventilación pulmonar (VE), el consumo de oxígeno $\left(\mathrm{VO}_{2}\right)$, la producción de dióxido de carbono $\left(\mathrm{VCO}_{2}\right)$, el $\mathrm{VO}_{2}$ máximo $\left(\mathrm{VO}_{2 \max }\right), \mathrm{VCO}_{2}$ máximo $\left(\mathrm{VCO}_{2 \max }\right)$. A partir de estas mediciones se determina de manera indirecta otras variables: los equivalentes ventilatorios para oxígeno $\left(\mathrm{VE} / \mathrm{VO}_{2}\right)$ y dióxido de carbono $\left(\mathrm{VE} / \mathrm{VCO}_{2}\right)$, y el cociente respiratorio. Además, se obtienen datos de la fuerza presora, el índice cronotrópico (9), y en algunas situaciones especiales se mide la saturación de pulso, durante la prueba y después del esfuerzo, por mencionar otras variables que se pueden determinar con la prueba $(10,11)$.

El consumo de oxígeno $\left(\mathrm{VO}_{2}\right)$ se define como el volumen de oxígeno $\left(\mathrm{O}_{2}\right)$ extraído del aire inhalado durante la ventilación pulmonar (VE) en un período de tiempo. En la práctica, el $\mathrm{VO}_{2 \text { pico }}$ es el valor más alto alcanzado, a pesar del aumento progresivo de la carga aplicada y suele considerarse como máximo $\left(\mathrm{VO}_{2 \text { maxx }}\right)$, cuando no se alcanzan criterios de maximidad, sin que en esencia representen lo mismo, pues este último representa la "máxima capacidad de una persona de absorber, transportar y consumir $\mathrm{O}_{2}$ " (4). Esto se calcula a partir de la diferencia entre el volumen de $\mathrm{O}_{2}$ en el aire inhalado y exhalado durante el ejercicio por unidad de tiempo. $\mathrm{El} \mathrm{VO}_{2}$ está determinado por la demanda de $\mathrm{O}_{2}$ celular y en personas sanas aumenta linealmente a medida que aumenta el trabajo externo (12).Por su parte, la producción de dióxido de carbono $\left(\mathrm{VCO}_{2}\right)$ es la diferencia entre el volumen de $\mathrm{CO}_{2}$ en el aire inhalado y exhalado durante el ejercicio por unidad de tiempo y representa la producción metabólica de dióxido de carbono, por tanto, refleja la capacidad del organismo para mantener el equilibrio de ácido básico (9). La $\mathrm{VCO}_{2}$ se ve afectada por los mismos factores que el $\mathrm{VO}_{2}$, sin embargo, es más dependiente de la ventilación debido a la mayor solubilidad del $\mathrm{CO}_{2}$ en la sangre (13).

Distintas variables en Pcpe, incluyendo el cociente respiratorio (RER) y los equivalentes ventilatorios, se derivan de la $\mathrm{VCO}_{2}(14)$. El RER expresa la relación entre la producción de $\mathrm{CO}_{2}$ y el consumo de $\mathrm{O}_{2}\left(\mathrm{VCO}_{2} / \mathrm{VO}_{2}\right)$. Actualmente es el mejor indicador no invasivo de la intensidad máxima o 
casi máxima del ejercicio. Los valores superiores a 1.0 pueden reflejar un ejercicio intenso, pero aquellos $\geq 1.10$ son los que busca una Pcpe (2) y han sido aceptados como un parámetro de agotamiento o casi agotamiento (15). La producción de ácido láctico cercano al agotamiento da como resultado un RER superior a 1, ya que se introduce $\mathrm{CO}_{2}$ adicional en el sistema a partir del tamponamiento con bicarbonato $\left(\mathrm{HCO}_{3}\right)$. Por lo tanto, un RER sustancialmente mayor que 1 en el ejercicio máximo es un marcador de esfuerzo máximo. De otra parte, la hiperventilación también puede causar un RER > 1 (14). Otros autores sostienen como criterios de que una persona ha alcanzado una prueba máxima cuando cumple al menos dos de los siguientes criterios: 1) alcanzar la meseta de $\mathrm{VO}_{2 \text { máx }}$ y que esta se mantenga, aunque la carga de trabajo se incremente; 2) alcanzar la frecuencia cardiaca máxima predicha y que el RER sea $\geq 1.15$ (16). En la figura 1 se presenta el comportamiento esperado del $\mathrm{VO}_{2}$ y la $\mathrm{VCO}_{2}$, los cuales van ascendiendo de manera lineal, siendo la $\mathrm{VO}_{2}$ mayor a la $\mathrm{VCO}_{2}$, hasta que se alcanza la meseta de $\mathrm{VO}_{2}$.

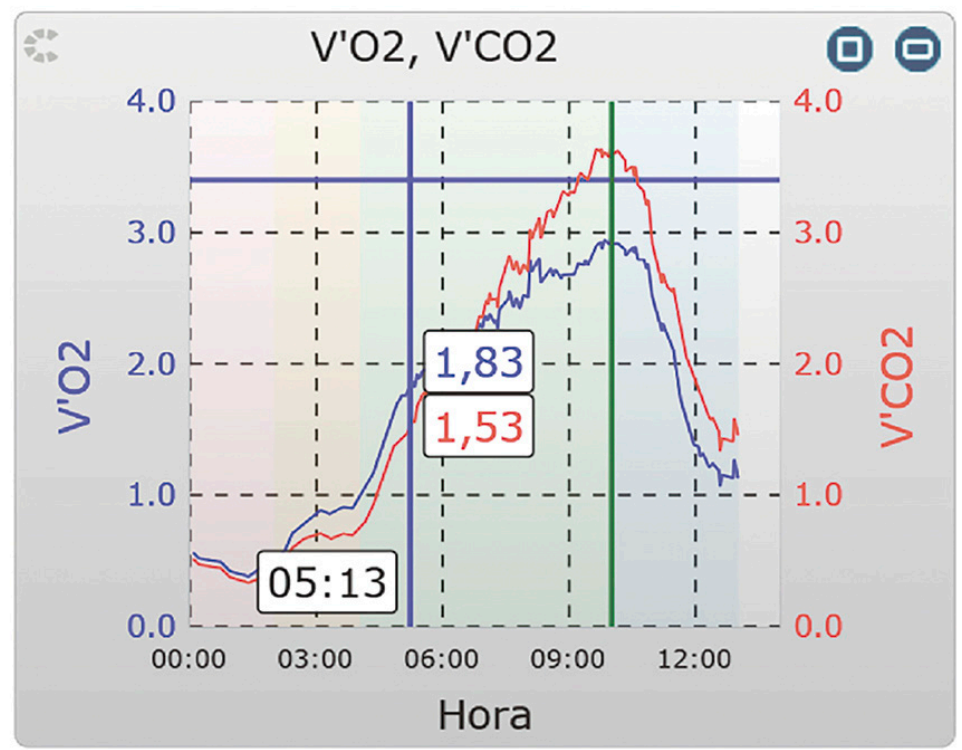

Figura 1. Gráfico del comportamiento del $\mathrm{VO}_{2} \mathrm{y} \mathrm{VCO}_{2}$. Fuente: Original de los autores. 
La ventilación pulmonar (VE) se expresa en litros por minuto y representa el volumen de aire que entra y sale de los pulmones. Se determina como el producto de la frecuencia respiratoria por el volumen corriente exhalado en cada ciclo y depende, en primer lugar, del proceso de respiración celular, en segundo lugar, de la interacción entre los sensores y receptores, que captan cambios físicos o químicos, en tercer lugar, del control central que gatilla la frecuencia y profundidad de cada respiración, enviando estímulos a los músculos respiratorios, que serán, en cuarto lugar, los efectores mecánicos que facilitarán la inspiración y la espiración. En tal sentido, se conoce que existen sensores ubicados a nivel del Sistena Nervioso Central (SNC), que captan cambios en el $\mathrm{pH}$ y la temperatura central. Por fuera del SNC se hallan los cuerpos carotideos y aórtico, sensibles a cambios de $\mathrm{PaCO}_{2}, \mathrm{PaO}_{2}$ y pH. También participan algunos receptores ubicados en la vía aérea superior (nariz, faringe y laringe) y los denominados receptores pulmonares (receptores de estiramiento, receptores de irritación y receptores yuxtacapilares). Finalmente, los receptores de los músculos respiratorios (usos neuromusculares y órgano tendinosos de Golgi), al condicionar el nivel de estiramiento y acortamiento de los músculos, también modulan la ventilación. En lo que respecta al control central, el centro neumotáxico ubicado en la Protuberancia, se encarga de inhibir la inspiración e incrementar la frecuencia respiratoria, mientras que el centro apnéustico, hace lo contrario, es decir, aumenta el tiempo inspiratorio. Los centros bulbares se encargan de estimular los músculos respiratorios (17).

En reposo, se ventilan de 7 a $9 \mathrm{~L} / \mathrm{min}$., de aire, pero en atletas ese valor puede alcanzar $200 \mathrm{~L} / \mathrm{min}$. con el esfuerzo máximo $(7,18)$. La ventilación aumenta continuamente durante el esfuerzo progresivo en Pcpe y experimenta aumentos adicionales influenciados por el metabolismo anaeróbico resultante de la acumulación de ácido láctico, bien definidos como los primeros y segundos umbrales de ventilación (19).

Por su parte, el pulso de oxígeno (pulso de $\mathrm{O}_{2}$ ) es la relación entre el $\mathrm{VO}_{2}$ y la frecuencia cardíaca (FC) y se expresa en $\mathrm{ml} /$ latido (20). Un pulso bajo de oxígeno durante el ejercicio puede indicar una disminución del volumen 
sistólico o una anomalía en la extracción de oxígeno del músculo esquelético. Una FC baja durante el ejercicio, causada por medicamentos beta-bloqueadores, puede elevar el pulso de $\mathrm{O}_{2}$ al disminuir el denominador (20).

Los equivalentes ventilatorios para oxígeno $\left(\mathrm{VE} / \mathrm{VO}_{2}\right)$ y para dióxido de carbono $\left(\mathrm{VE} / \mathrm{VCO}_{2}\right)$ son indicadores de eficiencia respiratoria. Se definen como las relaciones entre la ventilación pulmonar y el consumo de oxígeno $\left(\mathrm{VE} / \mathrm{VO}_{2}\right)$ o entre la ventilación pulmonar y la producción de dióxido de carbono $\left(\mathrm{VE} / \mathrm{VCO}_{2}\right)$, durante una prueba de ejercicio incremental ( 7 , 21). Ambas disminuyen desde el reposo hasta las intensidades de ejercicio submáximas y alcanzan valores mínimos antes del umbral anaeróbico (AT), cuando se produce un aumento progresivo, causado por el aumento de la ventilación para eliminar la producción adicional de $\mathrm{CO}_{2}$, que da lugar a un tampón de lactato por bicarbonato en sangre (6). En la figura 2 se presenta un ejemplo gráfico del comportamiento del $\mathrm{VE} / \mathrm{VO}_{2} \mathrm{y}$ del VE / $\mathrm{VCO}_{2}$, en el cual se observa el aumento paulatino del primero y descenso progresivo del segundo.

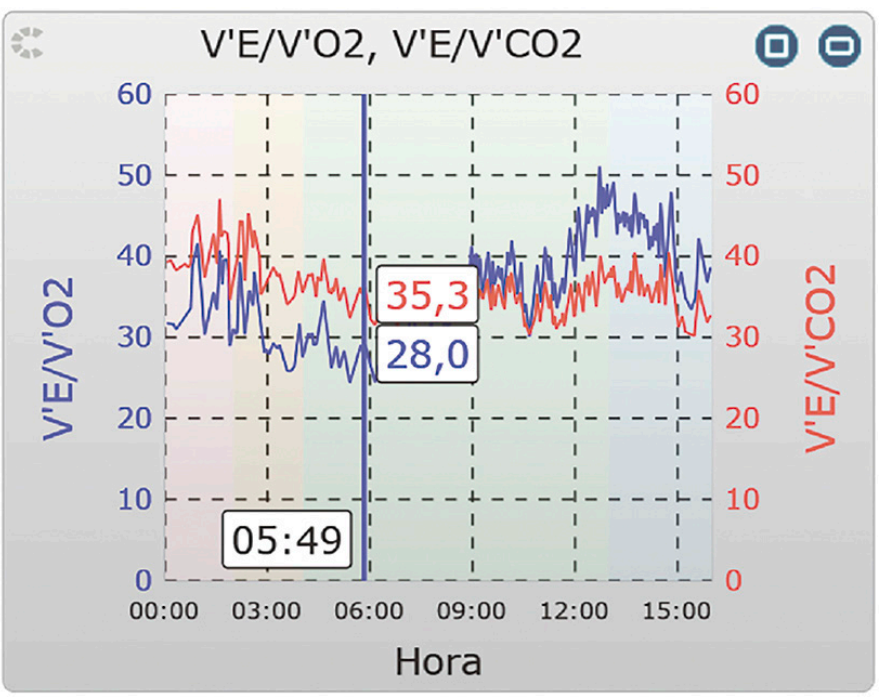

Figura 2. Gráfico del comportamiento del $\mathrm{VE} / \mathrm{VO}_{2} \mathrm{y}$ del $\mathrm{VE} / \mathrm{VCO}_{2}$ Fuente: Original de los autores. 
En el ámbito deportivo, la Pcpe es una prueba útil y valiosa para evaluar a los atletas y monitorear su progreso ante diferentes tipos de entrenamiento. Esta situación no resulta ajena en los militares, quienes deben tener un buen estado físico que les permita un rendimiento óptimo para cumplir con los requisitos operacionales, en ambientes estresantes, rigurosos y en condiciones de riesgo vital asociadas con su trabajo (22). Por lo tanto, históricamente las Fuerzas Armadas han invertido en el desarrollo de atletas de élite y nivel olímpico en todo el mundo (23), pero existe poca evidencia de Pcpe en la población de atletas militares y, a nuestro entender, este es el único estudio que se centra en este tipo de análisis en Colombia. Por lo tanto, esta investigación tuvo como objetivo analizar los equivalentes ventilatorios y el pulso de oxígeno máximos en la fase precompetitiva de un grupo de deportistas militares.

\section{Metodología}

Estudio observacional en el que se analizaron retrospectivamente 76 ergoespirometrías de deportistas militares de diferentes disciplinas (velocidad, fondo, orientación, triatlón y pentatlón, fútbol y baloncesto) en la fase precompetitiva de 2018. Solo se tuvieron en cuenta 60 pruebas que fueron máximas (cociente respiratorio $\geq 1,10)(24)$. Todos los sujetos fueron valorados en el Centro de Investigación de la Cultura Física (Cicfi) de la Escuela Militar de Cadetes "General José María Córdova" (Esmic), en tapiz rodante marca HP Cosmos y con Ergoespirómetro Metalyzer 3B-R2, a una temperatura de $22,6{ }^{\circ} \mathrm{C}$ y una presión barométrica de $560 \mathrm{mmHg}$. Todos debían haber realizado la prueba con protocolo de potencia aeróbica, iniciando con cuatro minutos de calentamiento a $4 \mathrm{~km} / \mathrm{h}$. Posteriormente, la velocidad inició a $7 \mathrm{~km} / \mathrm{h}$ y aumentó una milla, cada minuto, hasta el agotamiento, con inclinación constante del $1 \%$. Como variables ventilatorias se tuvo en cuenta la ventilación (VE), la producción de dióxido de carbono $\left(\mathrm{VCO}_{2}\right)$, el equivalente ventilatorio máximo para oxígeno y para dióxido de carbono, el pulso de oxígeno máximo $\left(\mathrm{VO}_{2 \text { máx }} / \mathrm{FC}_{\text {máx }}\right)$ el cociente respiratorio $\left(\mathrm{VCO}_{2}\right.$ 
/ $\left.\mathrm{VO}_{2}\right)$, la frecuencia respiratoria máxima $\left(\mathrm{FR}_{\text {máx }}\right)$, el volumen corriente máximo $\left(\mathrm{VC}_{\text {máx }}\right)$ y el consumo de oxígeno $\left(\mathrm{VO}_{2 \text { maxx }}\right)$. Dentro de las variables cardiacas, se analizó la frecuencia cardiaca máxima $\left(\mathrm{FC}_{\text {máx }}\right)$.

Los datos fueron agrupados en una matriz de Excel para analizarlos con el paquete estadístico SPSS 21, aplicando estadística descriptiva. La homogeneidad de la varianza se evaluó mediante la prueba de Levene. La distribución de los datos se verificó mediante la prueba de Shapiro-Wilk. Se aplicó la T de Student y el coeficiente de Pearson. El uso de los datos fue aprobado por el comité de ética de la Esmic, mediante acta Número10058, folio 209 de 30 de julio de 2019.

\section{Resultados}

Sesenta sujetos fueron analizados retrospectivamente. En la muestra 80 $\%(\mathrm{n}=48)$ fueron hombres y $20 \%(\mathrm{n}=12)$ mujeres, con edad promedio de $21 \pm 2$ ańos, peso $68 \pm 10 \mathrm{~kg}$, talla $1,71 \pm 10 \mathrm{~m}$. El Índice de Masa Corporal (IMC) promedio fue de $23,1 \pm 1,8 \mathrm{~kg} / \mathrm{m}^{2}$ con una superficie corporal de 1,8 $\pm 0,18 \mathrm{~m}^{2}, \mathrm{FC}_{\text {máx. }} 184 \pm 9 \mathrm{~L} / \mathrm{min}$.; $\mathrm{VO}_{\text {2máx. }} 45,6 \pm$ 6,6 ml / kg / min., $\mathrm{VO}_{\text {2máx }}$ $3,11 \pm 0,63 \mathrm{~L}$. Asimismo, el $\mathrm{VCO}_{2 \max .}$ fue 3,6 $\pm 0,7 \mathrm{~L}$, el VE máx $_{148,2} \pm 27$ $\mathrm{L}$, el VE $/ \mathrm{VO}_{2 \text { máx }} 52,6 \pm 10,2$. Por su parte, el VE/VCO ${ }_{2 \text { máx }}$ fue $41,17 \pm 4,6$, la $\mathrm{FR}_{\text {máx }} \cdot 68,9 \pm 10 \mathrm{r} / \mathrm{min}$, y el VC $\mathrm{máx} 2,1 \pm 0,48 \mathrm{~L}$. El Pulso de oxígeno fue de $17 \pm 3,5 \mathrm{ml} /$ latido y el cociente respiratorio de $1,2 \pm 0,10$. El $10 \%(\mathrm{n}=$ 6) de los deportistas eran de pentatlón, $22 \%(\mathrm{n}=13)$ de fútbol, $12 \%(\mathrm{n}$ = 7) de fondo, $20 \%(\mathrm{n}=12)$ de orientación, $8 \%(\mathrm{n}=5)$ de triatlón, 16 $\%(\mathrm{n}=10)$ de velocidad y $12 \%(\mathrm{n}=7)$ de baloncesto. Las diferencias por género se presentan en la tabla 1. En la tabla 2 se muestran las diferencias por modalidad deportiva. 
Tabla 1. Comportamiento de las variables cardiopulmonares por género

\begin{tabular}{|c|c|c|c|}
\hline \multirow[t]{2}{*}{ Variable } & \multicolumn{2}{|c|}{ Media y Ds. } & \multirow[t]{2}{*}{$\mathbf{p}^{*}$} \\
\hline & Hombre & Mujer & \\
\hline Edad (años) & $20,7 \pm 1,7$ & $20 \pm 1,9$ & 2,01 \\
\hline Peso (kg) & $71,19 \pm 8,5$ & $56,07 \pm 6,4$ & 0,00 \\
\hline Talla (m) & $1,74 \pm 0,08$ & $1,58 \pm 0,05$ & 0,00 \\
\hline $\operatorname{IMC}\left(\mathrm{Kg} / \mathrm{m}^{2}\right)$ & $23,37 \pm 1,85$ & $22,24 \pm 1,58$ & 0,05 \\
\hline Duración prueba (minutos) & $15,39 \pm 2,96$ & $14,28 \pm 3,14$ & 0,25 \\
\hline Superficie corporal $\left(\mathrm{m}^{2}\right)$ & $1,85 \pm 0,15$ & $1,57 \pm 0,12$ & 0,00 \\
\hline Velocidad (Km / h) & $18,83 \pm 1.63$ & $15,66 \pm 1,06$ & 0,00 \\
\hline FC Reposo (lpm) & $79 \pm 10$ & $83 \pm 8$ & 0,14 \\
\hline $\mathrm{FC}_{\text {máx. }}(\mathrm{lpm})$ & $183,5 \pm 8$ & $186,8 \pm 12$ & 0,26 \\
\hline $\mathrm{VO}_{2 \text { máx. }}(\mathrm{L})$ & $3,34 \pm 0,48$ & $2,21 \pm 0,26$ & 0,00 \\
\hline $\mathrm{VO}_{2 \text { máx }}(\mathrm{ml} / \mathrm{kg} / \mathrm{min})$ & $47,16 \pm 6,15$ & $39,66 \pm 4,9$ & 0,00 \\
\hline $\mathrm{VCO}_{2 \max } .(\mathrm{L} / \mathrm{min})$ & $3,89 \pm 0,48$ & $2,56 \pm 0,32$ & 0,00 \\
\hline $\mathrm{VE}_{\text {máx }} \cdot(\mathrm{L} / \mathrm{min})$ & $157,53 \pm 20,33$ & $111,3 \pm 17,98$ & 0,00 \\
\hline $\mathrm{VE} / \mathrm{VO}_{2 \text { máx. }}$ & $51,32 \pm 10$ & $57,90 \pm 9,85$ & 0,45 \\
\hline $\mathrm{VE} / \mathrm{VCO}_{2 \text { máx. }}$ & $40,62 \pm 4,52$ & $43,36 \pm 4,58$ & 0,66 \\
\hline $\mathrm{FR}_{\text {máx. }}(\mathrm{rpm})$ & $70,22 \pm 9,94$ & $64 \pm 9,34$ & 0,05 \\
\hline $\mathrm{VC}_{\text {máx. }}(\mathrm{L} / \mathrm{min})$ & $2,27 \pm 0,36$ & $1,75 \pm 0,27$ & 0,00 \\
\hline Pulso $\mathrm{O}_{2}$ (ml / latido) & $18,25 \pm 2,77$ & $12,08 \pm 1,44$ & 0,00 \\
\hline Cociente respiratorio & $1,20 \pm 0,11$ & $1,16 \pm 0,1$ & 0,23 \\
\hline
\end{tabular}

* Nivel de significancia $(\mathrm{p}<0,05)$.

Fuente: Original de los autores. 
Tabla 2. Comportamiento de las variables cardiopulmonares por modalidad deportiva.

\begin{tabular}{|c|c|c|c|}
\hline \multirow[t]{2}{*}{ Variable } & \multicolumn{2}{|c|}{ Media y Ds. } & \multirow[t]{2}{*}{$\mathbf{p}^{*}$} \\
\hline & Individual & Grupal & \\
\hline Edad (años) & $21 \pm 2$ & $21 \pm 1$ & 0,54 \\
\hline Peso (kg) & $64,9 \pm 8,4$ & $74,6 \pm 10,4$ & 0 \\
\hline Talla (m) & $1,70 \pm 0,1$ & $1,80 \pm 0,1$ & 0,01 \\
\hline $\operatorname{IMC}\left(\mathrm{Kg} / \mathrm{m}^{2}\right)$ & $23,0 \pm 1,9$ & $23,4 \pm 1,7$ & 0,43 \\
\hline Duración prueba (minutos) & $15,9 \pm 3,3$ & $13,8 \pm 1,5$ & 0,11 \\
\hline Superficie corporal $\left(\mathrm{m}^{2}\right)$ & $1,7 \pm 0,2$ & $1,9 \pm 0,2$ & 0 \\
\hline Velocidad (Km /h) & $18,3 \pm 2.3$ & $18,1 \pm 1,4$ & 0,74 \\
\hline FC. Reposo (lpm) & $80,5 \pm 10,8$ & $78,9 \pm 9,7$ & 0,57 \\
\hline $\mathrm{FC}_{\text {máx. }}(\mathrm{lpm})$ & $186,6 \pm 9,2$ & $179,4 \pm 7,5$ & 0,03 \\
\hline $\mathrm{VO}_{2 \text { máx }} .(\mathrm{L})$ & $3,0 \pm 0,7$ & $3,3 \pm 0,5$ & 0,18 \\
\hline $\mathrm{VO}_{2 \text { máx. }}(\mathrm{ml} / \mathrm{kg} / \mathrm{min})$ & $46,6 \pm 7,5$ & $43,9 \pm 3,9$ & 0,13 \\
\hline $\mathrm{VCO}_{2 \max .}(\mathrm{L} / \mathrm{min})$ & $3,4 \pm 0,7$ & $4 \pm 0,6$ & 0,01 \\
\hline $\mathrm{VE}_{\text {máx. }}(\mathrm{L} / \mathrm{min})$ & $144,7 \pm 28,3$ & $155,4 \pm 23,9$ & 0,15 \\
\hline $\mathrm{VE} / \mathrm{VO}_{2 \text { máx. }}$ & $55,5 \pm 8,5$ & $47 \pm 11,3$ & 0,02 \\
\hline $\mathrm{VE} / \mathrm{VCO}_{2 \text { máx. }}$ & $42,4 \pm 4,6$ & $38,4 \pm 3,6$ & 0.02 \\
\hline $\mathrm{FR}_{\text {máx. }}(\mathrm{rpm})$ & $70,9 \pm 11,1$ & $65,1 \pm 6$ & 0,03 \\
\hline $\mathrm{VC}_{\text {máx. }}(\mathrm{L} / \mathrm{min})$ & $2,1 \pm 0,4$ & $2,4 \pm 0,4$ & 0,01 \\
\hline Pulso $\mathrm{O}_{2}$ (ml / latido) & $16,4 \pm 3,7$ & $18,4 \pm 3$ & 0,03 \\
\hline Cociente Respiratorio & $1,18 \pm 0,1$ & $1,2 \pm 0,1$ & 0,04 \\
\hline
\end{tabular}

* Nivel de significancia $(\mathrm{p}<0,05)$.

Fuente: Original de los autores.

Los $\mathrm{VE} / \mathrm{VO}_{2 \text { máx. }}$ y VE / $\mathrm{VCO}_{2 \text { máx. }}$ no mostraron diferencias significativas por género, pero sí por modalidad deportiva. Deporte individual VE / $\mathrm{VO}_{2 \text { máx. }} 55,5 \pm 8,5$ vs $47 \pm 11,3$ en deporte en equipo y $\mathrm{VE} / \mathrm{VCO}_{2 \text { max. }} 42,4$ $\pm 4,6$ vs $38,4 \pm 3,6$, respectivamente, con $\mathrm{p}(0,00)$ en ambos casos. 
$\mathrm{El} \mathrm{PulO}_{2 \text { max. }}$ evidenció diferencias por género. Hombres: 18,25 $\pm 2,77$ $\mathrm{ml} /$ latido y $12,08 \pm 1,44 \mathrm{ml} /$ latido en mujeres, $p(0,00)$. También por modalidad deportiva: $16,4 \pm 3,7 \mathrm{ml} /$ latido en deporte individual y 18,4 $\pm 3 \mathrm{ml} /$ latido en deporte por equipo, $p(0,01)$. El VE / $\mathrm{VCO}_{2 \text { máx. }}$ se relacionó con el VE/ $\mathrm{VO}_{2 \text { máx. }}(\mathrm{r}=549, \mathrm{p}=0,00)$, con el $\mathrm{VO}_{2 \text { máx. }}(0,342 \pm 0,00)$, con el tiempo de duración de la prueba $(\mathrm{r}=0,385, \mathrm{p}=0,00)$. A su vez, la duración de la prueba se relacionó con el $\mathrm{VO}_{2 \text { máx }}(\mathrm{r}=0,518, \mathrm{p}=0,00)$. La $\mathrm{VE} / \mathrm{VO}_{2 \text { máx. }}$ se relacionó con el peso $(\mathrm{r}=-373, \mathrm{p}=0,00)$, con el Índice de Masa Corporal $(r=-317, p=0,00)$ y con la $\mathrm{VCO}_{2 \text { máx. }}(r=-317, \mathrm{p}=0,00)$. El $\mathrm{PulO}_{2 \text { máx. }}$ se relacionó con la talla $(\mathrm{r}=0,693, \mathrm{p}=0,00)$, con el peso $(\mathrm{r}=$ 732, $\mathrm{p}=0,00)$, con el IMC $(\mathrm{r}=335, \mathrm{p}=0,00)$, con la velocidad $(\mathrm{r}=414$, $\mathrm{p}=0,00)$, con la $\mathrm{VCO}_{2 \text { máx. }}(\mathrm{r}=0,781, \mathrm{p}=0,00)$, con la $\mathrm{VE}_{\text {máx. }}(\mathrm{r}=0,828, \mathrm{p}$ $=0,00)$ y con el $\mathrm{VO}_{2 \text { máx. }}(\mathrm{r}=0,661, \mathrm{p}=0,00)$. $\mathrm{El} \mathrm{VO}_{2 \text { máx. }}$ se relacionó con la $\mathrm{FR}_{\text {máx. }}(\mathrm{r}=0,474, \mathrm{p}=0,00)$.

\section{Discusión}

En la fase precompetitiva, el principal objetivo es evaluar las condiciones físicas y funcionales de los deportistas, con el fin de implementar planes de entrenamiento que optimicen el desempeño durante la competencia. En el presente trabajo se evidenció que el equivalente ventilatorio máximo para dióxido de carbono, el consumo de oxígeno máximo y el pulso de oxígeno fueron variables que tuvieron un impacto significativo en la duración de la prueba y, por tanto, en el rendimiento de los deportistas valorados.

En relación con lo descrito, se ha informado que el VE / $\mathrm{VCO}_{2}$ no tiene relación con la capacidad del atleta para usar oxígeno o alcanzar un alto rendimiento (25). No obstante, en este estudio se halló una correlación positiva entre esta variable medida en el esfuerzo máximo y la duración de la prueba en la fase precompetitiva, lo cual sugiere una asociación entre la ventilación y el rendimiento. Por esta razón se recomienda monitorearla en el entrenamiento, como lo sugieren Sauer, Pérez y Cartelli (26), quienes sostienen que el equivalente ventilatorio para dióxido de carbono puede ser 
medido en las diferentes fases de la ergoespirometría, lo cual implica que pueda ser medido en el momento en que se alcanza el $\mathrm{VO}_{2 \text { máx. }}$, como se realizó en este estudio De hecho, en varios trabajos en los que se compara la efectividad de diferentes tipos de entrenamiento sobre variables cardiopulmonares, se analiza el efecto sobre el $\mathrm{VE} / \mathrm{VCO}_{2}$, medido en el máximo esfuerzo $(27,28)$, en el entendido de que como no existe un consenso que especifique en qué momento debe evaluarse (29), siempre será un indicativo de la eficiencia para ventilar. En este punto es necesario recordar que por medio de la ventilación el sujeto puede ingresar oxígeno y eliminar dióxido de carbono y que según el comportamiento de la curva de disociación de la hemoglobina, la disminución del $\mathrm{pH}$ plasmático, el aumento de la $\mathrm{PaCO}_{2}$ o de la temperatura, entre otros, provocan una disminución de la afinidad de la hemoglobina por el oxígeno, favoreciendo su entrega a los tejidos (30), situación que podría explicar la relación que se halló en la fase máxima en los deportistas valorados.

En el mismo orden de ideas, el trabajo de Nalbandiano y colaboradores (31), que también consideraron la duración de la prueba como un indicador de rendimiento, documentó en un grupo de diez hombres deportistas que ni el $\mathrm{VO}_{2 \text { máx. }}$ ni la resistencia son influidos por la frecuencia respiratoria. Contrario a ello, en este trabajo se evidenció una relación significativa entre el $\mathrm{VO}_{2 \text { máx. }}$ y la $\mathrm{FR}_{\text {máx }}$. y aunque no se halló relación entre la $\mathrm{FR}$ y la duración de la prueba, es posible pensar que, si la $\mathrm{FR}$ se relaciona con el $\mathrm{VO}_{2 \text { máx.' }}$ también lo haga sobre el rendimiento, aspecto que podría abordarse en otro estudio. En este caso, el incremento exagerado de la frecuencia respiratoria en la fase máxima hace que el volumen corriente se reduzca y por consiguiente se incremente la $\mathrm{PaCO}_{2}$, lo cual genera, como lo describe Minas desde la fisiología, que el efecto Bohr se potencie y por lo tanto optimice la entrega de $\mathrm{O}_{2}(32,33)$. Esta situación también explicaría la relación hallada entre el VE / $\mathrm{VO}_{2}$ y la $\mathrm{VCO}_{2}$ y entre el VE / $\mathrm{VCO}_{2}$ y el $\mathrm{VO}_{2}$.

Por su parte, Herdy y Uhlendorf establecieron baremos de $\mathrm{VO}_{2 \text { máx. }}$ en 2.388 hombres y 1.534 mujeres brasileños sanos, sedentarios y físicamente activos, con valores promedio en la población activa de 50,6 \pm 7,3 $\mathrm{ml} / \mathrm{kg}$ / 
min. para los hombres y 38,9 $\pm 5,7 \mathrm{ml} / \mathrm{kg} / \mathrm{min}$. para las mujeres (4). En el presente estudio se encontraron valores levemente superiores en las mujeres y levemente inferiores en los hombres, diferencias que se pueden deber al tipo de población, la raza y la edad, pues fue en el grupo de 15 a 24 años de Herdy y Uhlendorf con los que se comparan nuestros resultados, mientras que en el presente estudio la edad mínima fue 18 años.

Asimismo, teniendo en cuenta que el $\mathrm{VO}_{2}$ depende, en parte, de una correcta difusión de gases, que la difusión de gases se ve influenciada por constantes de tiempo (34) y que en actividades intensas de alta demanda metabólica la FC llega a niveles máximos que acortan el tiempo de exposición del glóbulo rojo en la membrana hematogaseosa, es posible explicar que las frecuencias máximas alcanzadas por los deportistas pudieron influir para que el $\mathrm{VO}_{2 \text { máx. }}$ estuviera disminuido, si se comparan los valores hallados en otros deportistas de las mismas edades en la fase precompetitiva (33). En este punto es importante referir como posible explicación de estos hallazgos la heterogeneidad de la población, en cuanto a modalidad, pues no todos los deportistas pertenecían a la misma disciplina y, por tanto, sus diferentes requerimientos metabólicos, aunque pertenecieran a la misma modalidad, influyen en la respuesta fisiológica al ejercicio (35). Adicionalmente, no se contó con datos que pudieran establecer cuánto tiempo llevaban practicando el deporte, de manera que algunos de ellos podrían ser aficionados, situación que también podría influir para que las diferencias de los equivalentes ventilatorios de $\mathrm{CO}_{2} \mathrm{y} \mathrm{O}^{2}$, entre las modalidades deportivas, hayan tenido el comportamiento registrado. Por esta razón se recomienda tener en cuenta estos aspectos en fututos estudios.

Finalmente, el trabajo de Padilla con deportistas de resistencia mexicanos documentó una relación positiva entre $\mathrm{PulO}_{2 \text { máx }} \mathrm{y} \mathrm{VO}_{2 \text { máx. }}$ absoluto, pero negativa entre $\mathrm{PulO}_{2 \text { máx }}$ y $\mathrm{FC}_{\text {máx. }}$ (20), hallazgos similares a los de este trabajo, lo cual corrobora que el entrenamiento, al generar cambios en el número de miofribrillas y en su capacidad oxidativa, hace que se requiera menor frecuencia cardiaca para lograr mejores valores de $\mathrm{VO}_{2}$ (36). Por ello el pulso de oxígeno complementa la evaluación cardiorrespiratoria, tal y como lo concluye el mismo Padilla (20). 


\section{Conclusiones}

El equivalente ventilatorio para dióxido de carbono máximo (VE / $\mathrm{VCO}_{2 \text { máx. }}$ ) influye en la duración de la prueba, por lo que el entrenamiento de la técnica de la respiración y o de los músculos respiratorios podrían influir en el desempeño deportivo.

El $\mathrm{PulO}_{2 \text { máx. }}$ permite identificar la resistencia cardiopulmonar y por tanto el desempeño deportivo. Esto se vio reflejado en su relación directa con la velocidad de los deportistas valorados. Por ello, el entrenamiento, además de potenciar la ventilación, debe procurar potenciar el $\mathrm{PulO}_{2 \text { máx. }}$ haciendo diferencias por género y tipo de deporte.

\section{Agradecimientos}

Los autores agradecen al Centro de Investigación de la Cultura Física (Cicfi) de la Esmic. Así mismo, al laboratorio cardiopulmonar y al servicio de Nutrición Deportiva por el adecuado registro de la información.

\section{Financiación}

Este estudio fue financiado por la Escuela Militar de Cadetes "General José María Córdova”. Convocatoria financiada 01 de 2019.

\section{Referencias}

1. Cid-Juárez S, Miguel-Reyes JL, Cortés-Téllez A, Gochicoa-Rangel L, Mora-Romero UJ, Silva-Cerón M, Torre-Bouscoulet L. Prueba cardiopulmonar de ejercicio. Recomendaciones y procedimientos de ejercicio. Neumol. Cir. Torax. 2015 julio; 74 (3): 207-221. Disponible en: https://www.medigraphic.com/pdfs/neumo/nt-2015/ nt153h.pdf

2. Thomas A, Burdiat G (Dres.). Pruebas de esfuerzo cardiopulmonar en la práctica clínica. Rev. Urug. Cardiol. 2010 junio; 25 (1): 17-27. Disponible en: http://www. scielo.edu.uy/pdf/ruc/v25n1/v25n1a04.pdf 
3. Balady GJ, Arena R, Sistsema K, Myers J, Coke L, Fletcher GF, et al. Clinician's guide to cardiopulmonary exercise testing in adults: a scientific statement from the DOI: http://dx.doi.org/10.1161/CIR.0b013e3181e52e69

4. Herdy AH, Unhlendorf D. Valores de referencias para el test cardiopulmonar para hombres y mujeres sedentarios y activos. Archivos Brasileiros de Cardiologia. 2011 noviembre; 96 (1): 54-59. https://doi.org/10.1590/S0066-782X2010005000155.

5. Gibbons RJ, Balady GJ, Beasley JW, Bricker JT, Duvernoy WJ, Froelicher VF, et al. ACC/AHA guidelines for exercise testing: a report of the American College of Cardiology/American Heart Association Task Force on Practice Guidelines. J Am. Coll. Cardiol. 1997 julio; 30 (1): 260-311. DOI: http://dx.doi.org/10.1016/s07351097(97)00150-2

6. Guazzi M, Adams V, Conraads V, Halle M, Mezzani A, Vanhees L, et al. European Association for Cardiovascular Prevention \& Rehabilitation; American Heart Association. EACPR/AHA Scientific Statement Clinical recommendations for cardiopulmonary exercise testing data assessment in specific patient populations. Circulation. 2012 octubre; 126 (18): 2261-2274. DOI: http://dx.doi.org/10.1161/ CIR.0b013e31826fb946

7. Herdy AH, Fonteles Ritt E, Stein R, Gil Soares de Araujo C, Milani M, Meneghelo RS, et al. Cardiopulmonary exercise test: Backgruond, Applicability and interpren tation. Arq. Bras. Cardiol. 2016 noviembre; 107 (5): 467-481. DOI: http://dx.doi. org/10.5935/abc.2016017

8. Takashi AD, Flavia B, Rocha ML, Kaminura MA, Mello MT, Tufik S, et al. Impacto del entrenamiento en el umbral ventilatorio sobre la capacidad cardiopulmonar y funcional en pacientes con sobrepeso con enfermedad renal crónica. J. Bras. Nefrol. 2012 junio; 34 (2): 139-147.

9. Ilarraza-Lomelí H. Prueba de ejercicio con análisis de gases espirados. Arch. Cardiol. Méx. 2012 abril / junio; 82 (2): 160-169. Disponible en: http://archivoscardiologia.com/previos/(2012)\%20ACM\%20Vol\%2082.\%202\%20ABRIL-JUNIO/ ACMX_2012_82_2_160-169.pdf

10. Arena R, Myers J, Guazzi M. The clinical and research applications of aerobic capacity and ventilatory efficiency in heart failure: an evidence-based review. Heart Fail Rev. 2008 junio; 13 (2): 245-269. DOI: http://dx.doi.org/10.1007/s10741-007-9067-5

11. Arena R, Lavie CJ, Milani RV, Myers J, Guazzi M. Cardiopulmonary exercise testing in patients with pulmonary arterial hypertension: an evidence-based review. J Heart Lung Trnasplant. 2010 febrero; 29 (2): 159-173. DOI: http://dx.doi.org/10.1016/j. healun.2009.09.003

12. López Chicharro J, López Mojarrez LM. Fisiología clínica del ejercicio. McArdle WD, Katch FI, Katch VL, editors. España: McGraw-Hill/Interamericana; 2004.

13. Illescas L, Kohn E, Bouchacourt JP, Cavalleri F, Hurtado J, Riva J. Utilidad de las medidas derivadas de la presión parcial de $\mathrm{CO} 2$ en pacientes adultos sometidos cirugía 
cardíaca con circulación extracorpórea. Rev. Chil. Anest. 2019 agosto; 48 (5): 412-426. Disponible en: https://doi.org/10.25237/revchilanestv48n05.06

14. Debapriya Datta EN, ZuWallack R. Cardiopulmonary exercise testing in the assessment of exertional dyspnea. Ann. Thorac. Med. 2015 junio; 10 (2): 77-86. DOI: http://dx.doi.org/10.4103/1817-1737.151438

15. Monteiro de Almeida AE, de Moraes Stefani C, do Nascimento A, de Almeida NM, da Cruz Santos A, Pinto Ribeiro J, Stein R. An equation for the prediction of oxygen consumption in a a brazilian population. Arq. Bras. Card. 2014 octubre; 103 (4): 299-307. Disponible en: https:/www.ncbi.nlm.nih.gov/pmc/articles/PMC4206360/

16. Hall-López JA, Ochoa-Martínez PY, Moncada-Jiménez J, Ocampo Méndez MA, Martínez García I, Martínez García MA. Confiabilidad del consumo máximo de oxigeno evaluado en pruebas de esfuerzo. Nutrición Hospitalaria. 2015 abril; 31 (4): 1726-1732. Disponible en: http://dx.doi.org/10.3305/nh.2015.31.4.8487

17. García Cabrera L, Rodríguez Reyes O, Rodríguez Carballosa OB. Regulación de la respiración: organización morfofuncional de su sistema de control. Medisan. 2011 abril; 15 (4): 558-567. Disponible en: http://scielo.sld.cu/scielo.php?script=sci_arttext\&pid=S1029-30192011000400020\&lng=es.

18. Wasserman K, Whipp BJ. Exercise physiology in health and disease. Am. Rev. Resp. Dis. 1975 agosto; 112 (2): 219-249. DOI: http://dx.doi.org/10.1164/arrd.1975.112.2.219

19. Corrá U, Giordano A, Bosimini E, Mezzani A, Piepoli M, Coats A, Giannuzzi P. Oscillatory ventilation during exercise in patients with chronic heart failure: clinical correlates and prognostic implications. Chest Journal. 2002 mayo; 121 (5):1572-1580. DOI: http://dx.doi.org/10.1378/chest.121.5.1572

20. Padilla Pérez J. Pulso de oxígeno y su cinética de la fase dos transitoria durante una prueba de esfuerzo máximo de VO2 en adultos mayores. Rev. Hosp. Jua. Mex. 2011; 78 (1): 6-11. Disponible en: https://www.medigraphic.com/pdfs/juarez/ju-2011/ ju111b.pdf

21. Arena R, Myers J, Hsu L, Peberdy MA, Pinkstaff S, Bensimhom D, et al. The minute ventilation/carbon dioxide production slope is prognostically superior to the oxygen uptake efficiency slope. J Card Fail. 2007 agosto; 13 (6): 462-469. DOI: http://dx.doi. org/10.1016/j.cardfail.2007.03.004

22. JoEllen M S, Burkhardt TA. Introduction to the Tactical Athlete Special Issue. J. Athl. Train. 2016 noviembre; 51 (11): 845. DOI: http://dx.doi.org/10.4085/1062-605051.12 .16

23. Xavier R, Sánchez C, Paulucio D, Moreira da Silva I, Velasque R, Noriega FS, et al. A multidimensional approach to assessing anthropometric and aerobic fitness profiles of Elite Brazilian Endurance Athletes and Military Personnel. Mil Med. 2019 diciembre; 184(11-12): 875-881. DOI: http://dx.doi.org/10.1093/milmed/usz003

24. Niño Hernández CA. Evaluación de la aptitud cardiorrespiratoria. Mov. Cient. 2010; 4 (1): 68-72. https://doi.org/10.33881/2011-7191.\%25x 
25. Salazar-Martínez E, Rodrigues de Matos T, Arrans P, Santalla A, Naranjo Orellana J. Ventilatory efficiency response is unaffected by fitness level, ergometer type, age, or body mass index in male athletes. Biology of Sport. 2018 diciembre; 35 (4):. 393-398. DOI: http://dx.doi.org/10.5114/biolsport.2018.78060

26. Sauer D, Perez A, Cartelli L. Efeito do treinamento sobre a eficiência ventilatória de indivíduos saudáveis. Rev. Bras. Med. Esporte. 2014 mayo; 20 (6): 470-473. https:// doi.org/10.1590/1517-86922014200601814

27. Salazar-Martínez E, Santalla A, Naranjo Orellana J, Strobl J, Burtscher M, Menz V. Influence of high-intensity interval training on ventilatory efficiency in Trained Athletes. Respiratory Physiology \& Neurology. 2018 abril; 250: 19-23. DOI: http:// dx.doi.org/10.1016/j.resp.2018.01.016

28. Di Paco A, Dubé B-P, Laveneziana P. Cambios en la respuesta ventilatoria al esfuerzo en deportistas entrenados: efectos beneficiosos sobre la fisiología respiratoria más allá del rendimiento cardiovascular. Arch. Bronconeumol. 2017; 53(5): p. 237-244. DOI: http://dx.doi.org/10.1016/j.arbres.2016.11.023

29. Salazar-Martínez E, Terrados N, Burtscher M, Santalla A., Naranjo Orellana J. Ventilatory efficiency and breathing pattern in world-class cyclists: A three-year observational study. Respiratory Physiology \& Neurobiology. 2016 abril; 229: 17-23. DOI: http://dx.doi.org/10.1016/j.resp.2016.04.001

30. Gutiérrez Muñoz FR. Insuficiencia respiratoria aguda. Acta Méd. Peruana. 2010 octubre / diciembre; 27 (4): 286-297. Disponible en: http://www.scielo.org.pe/scielo. php?script=sci_arttext\&pid=\$1728-59172010000400013\&lng=es

31. Nalbandiano M, Radak Z, Taniguchi J. How different respiratory patterns affect cardiorespiratory variables and performance. Int. J. Exerc. Sci. 2017 mayo; 10 (3): 322-329.

32. Patel AK, Cooper JS. Physiology, Bohr Effect. In StatPearls [Internet]. Treasure Island(FL): StatPearls Publishing; 2019.

33. Di Paco A, Dubé BP, Laveneziana P. Changes in Ventilatory Response to Exercise in Trained Athletes: Respiratory Physiological Benefits Beyond Cardiovascular Performance. Arch. Bronconeumol. 2017 mayo; 53 (5): 237-244. DOI: http://dx.doi. org/10.1016/j.arbres.2016.11.023

34. Méndez J E, Zeledon SS, Zamora L F, Cortés V A. Un acercamiento a la cinética del oxígeno. Rev. Costarric. Cardiol. 2004 mayo; 6 (2): 37-42. Disponible en: http://www. scielo.sa.cr/scielo.php?script=sci_arttext\&pid=\$1409-41422004000100006\&lng=en

35. Martínez - Sanz JM, Urdampilleta A. Necesidades nutricionales y planificación dietética en deportes de fuerza. Motricidad. European Journal of Human Movement. 2012 diciembre; 29: 95-114. Disponible en: https://www.redalyc.org/ pdf/2742/274224827007.pdf

36. Fernández-Lázaro D, Díaz J, Caballero A, Córdova A. Entrenamiento de fuerza y resistencia en hipoxia: efecto en la hipertrofia muscular. Biomédica. 2019 septiembre; 39 (1): 212-220. https://doi.org/10.7705/biomedica.v39i1.4084 